\title{
Estudo sobre um surto de malária entre os índios Mayongong e Sanomã (Norte de Roraima) $\left(^{*}\right)$
}

\author{
José João Ferraroni ( ${ }^{(*)}$ ) \\ Jack Hayes ( ${ }^{* *}$ )
}

\begin{abstract}
Resumo
Em abril de 1977 ocorreu um surto de malária falciparum nas tribos Sanomă e Mayongong, em Roraima, na fronteira do Brasil e Venezuela. Duzentos e sessenta e oito silvícolas foram examinados para malária e 91 casos foram positivos, dois para Plasmodium vivax e 89 para Plasmodium falciparum. $\mathrm{O}$ vetor responsável pela epidemia foi o Anopheles darlingi com uma atividade média de 64 picadas/homem por noite. Três casos de falciparum foram resistentes às cloroquinas, respondendo favoravelmente ao Fansidar (sulfadoxina e pirimetamina). Houve diferença distinta nas duas tribos baseada na proximidade da populacão do vetor. Essas diferenças foram apresentadas por idade e sexo. Foi interessante a descoberta de um grupo de Sanomá que, no esforço para livrar-se ao ataque da malária, fugiu para outra área. Este grupo foi encontrado em localidade que não possuia mosquitos.
\end{abstract}

\section{INTRODUÇÃo}

Um surto de malária ocorreu em abril de 1977 no acampamento indígena do Uauaris $\left(04^{\circ} \mathrm{N} 08^{\prime} \mathrm{N}, 064^{\circ} \mathrm{W} 029^{\prime} \mathrm{W}\right)$ na região noroeste do Brasil (Roraima), fronteira com a Venezuela. Lá existem dois grupos de índios: os Sanomã, parte do grupo Yanomama, e os Mayongong, dos Yekuana ou Maquiritari. Estas duas tribos têm uma coexistência de muitos anos, a maioria na Venezuela (Wilbert, 1963). Os Mayongong no Brasil, situados no Uauaris são conhecidos há mais de 75 anos.

O primeiro contato permanente com esses índios foi feito em 1964 pela MEVA (Missão Evangélica Vale do Amazonas). Esses missionários construíram moradias no local e têm mantido residência desde abril de 1965 . Nessa époça houve uma epidemia de malária, 100 amosiras de sangue (em lâmina) foram enviadas para a CEM (atualmente SUCAM) das quais noventa foram positivas (predominando a infecção pelo Plasmodium falciparum) e cinco indios morreram. Os missionários usaram. no tratamento, cloroquina (Aralen) e amodiaquina (Camoquin); a borrifação das casas com DDT foi realizada com cooperação da CEM (Borgman, 1977). Um indio idoso recaiu com malária e houve alguns casos esporádicos, mas não como em 1965. Com exceção desta epidemia, não houve nenhuma após 1965: aiguns casos esporádicos de infecção malárica têm ocorrido na tribo dos Mayongong, principalmente quando regressam de suas viagens da Venezuela.

O posto central dos missionários está localizado próximo a uma pista de pouso, construída na tribo Sanomã: três quilômetros rio abaixo está localizada a aldeia dos Mayongong.

Cinco dias antes da equipe de pesquisadores chegar à área, um segundo grupo de Sanomã construiu um acampamento temporário há uns 100 metros de distância da aldeia dos Mayongong. Isto foi feito para receberem melhor assistência médica dos missionários. Este grupo vivia há 10 quilômetros rio abaixo da pista de pouso.

\section{MATERIAIS E MÉtodos}

Amostras de sangue foram obtidas por venóclise ou por punção digital. Foram feitos esfregaços e gota espessa em lâminas, que foram fixadas e coradas pelo método de Giemsa de acordo com os padrões da OMS (OMS, 1975). O diagnóstico foi dado examinando as lâminas com um aumento de 1000 vezes. Foram coletados mosquitos dos índios, deitados em redes, durante as 24 horas do dia, usando as-

\footnotetext{
(*) - Pesquisa no 2017/102, do INPA. Patrocinada pelo Conselho Nacional de Desenvolvimento Cientifico e Tecnológico (CNPq.).

$\left({ }^{*}\right)$ - Instituto Nacional de Pesquisas da Amazônia, Manaus.
} 
pirador oral e, durante a noite laterna a pilha. Os mosquitos foram identificados com ajuda de lupas e microscópio binocular. Mosquitos picando o homem foram também coletados no bosque próximo a aldeia dos Mayongong e em uma barraca, construída temporariamente, no centro da aldeia.

\section{Resultados}

Os dados dos índios examinados em cada tribo e a taxa de infecção por idade e sexo estão representadas no gráfico I (Sanomã) e no gráfico II (Mayongong). As tabelas I e II dão os resultados das lâminas positivas para ambas as tribos. Somente dois casos de infecção por Plasmodium vivax foram encontrados. En- contramos 89 casos de infecção por $P$. falciparum. Em cinco índios encontramos somente gametócitos de $P$. falciparum, indicando infeccões bem estabelecidas.

Não foram coletadas amostras de sangue de pessoas com idade inferior a um ano, a não ser que apresentassem sintomas de malária. Um total de 156 lâminas foi obtido da tribo dos Mayongong e 112 dos Sanomã. Foi muito dificil ccletar mosquitos na aldeia dos Sanomã, pois este grupo se tinha mudado para uma maloca recém-construída, para evitar "espíritos contaminantes" da velha residência. Muitos índios viviam juntos, superlotando uma maloca, onde foi difícil coletar mosquitos devido a sua baixa freqüência.

TABELA 01

Tribo Sanomã, total de pessoas examinadas, positivos e negativos, relacionando faixa etária e sexo.

\begin{tabular}{|c|c|c|c|c|c|c|c|c|c|c|c|c|c|c|}
\hline \multicolumn{8}{|c|}{ MASCULINO } & \multicolumn{7}{|c|}{ FEMININO } \\
\hline \multirow{2}{*}{$\begin{array}{l}\text { IDADE } \\
\text { (ANOS) }\end{array}$} & \multicolumn{6}{|c|}{ PARASITEMIA } & \multirow{2}{*}{ TOTAL } & \multicolumn{6}{|c|}{ PARASITEMIA } & \multirow{2}{*}{ TOTAL } \\
\hline & $+/ 2$ & + & ++ & +++ & FG & NEG & & $+/ 2$ & + & ++ & +++ & FG & NEG & \\
\hline Até- 04 & 01 & 01 & & & & 07 & 09 & & & 03 & & & 03 & 06 \\
\hline $05-09$ & 01 & & 01 & & 01 & 07 & 10 & & 02 & 01 & & & 11 & 14 \\
\hline $10-14$ & & & & & & 05 & 05 & & & 01 & & & 04 & 05 \\
\hline $15-19$ & & 01 & 02 & & & 04 & 07 & 01 & & 02 & & & 04 & 07 \\
\hline $20-24$ & & & 02 & 01 & & 10 & 13 & & & 01 & & & 03 & 04 \\
\hline $25-29$ & & & & & & 03 & 03 & & & & & & 03 & 03 \\
\hline $30-34$ & & & & & & 01 & 01 & & & 02 & & & 03 & 05 \\
\hline $35-39$ & & & & & 01 & 04 & 05 & & & & & & 01 & 01 \\
\hline $40-44$ & & & & & & 01 & 01 & & 01 & 01 & & & 04 & 06 \\
\hline $45-49$ & & & & & & 01 & 01 & & & & & & & 00 \\
\hline $50-54$ & & & & & & 03 & 03 & & & & & & & 00 \\
\hline $55-59$ & & & & & & & 00 & 01 & & & & & & 01 \\
\hline $60-64$ & & & & & & & 00 & & & & 01 & & 01 & 02 \\
\hline TOTAL & 02 & 02 & 05 & 01 & 02 & 46 & 58 & 02 & 03 & 11 & 01 & 00 & 37 & 54 \\
\hline
\end{tabular}

\footnotetext{
$+/ 2$ - $<01$ parosito por campo mil $X$ segundo o critério da OMS.

$+\quad-02$ parositos por compo mil $\times$ segundo o critério da OMS.

$++\quad 03 \%$ a 20 parasitas por campo mil $\times$ segundo o critério da OMS.

+++ - 021 a 200 parasitas por campo mil $X$ segundo o critério da OMS.

FG - Somente forma gametócita no songue periférico.

NEG - Resultado Negativo.
} 


\section{SANOMĀ - UAUARIS}

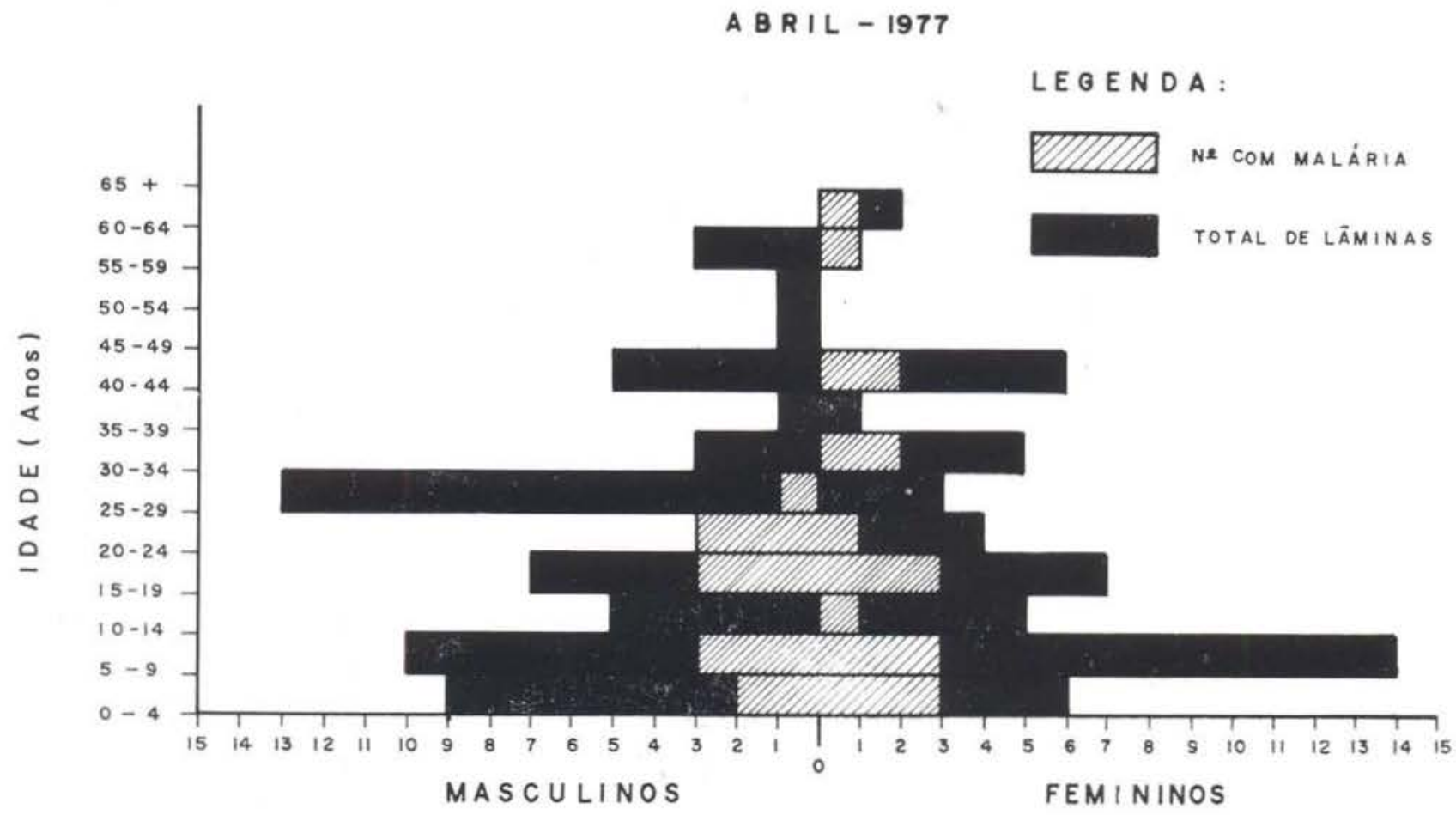

MAYOGONG - UAUARIS

ABRIL - 1977

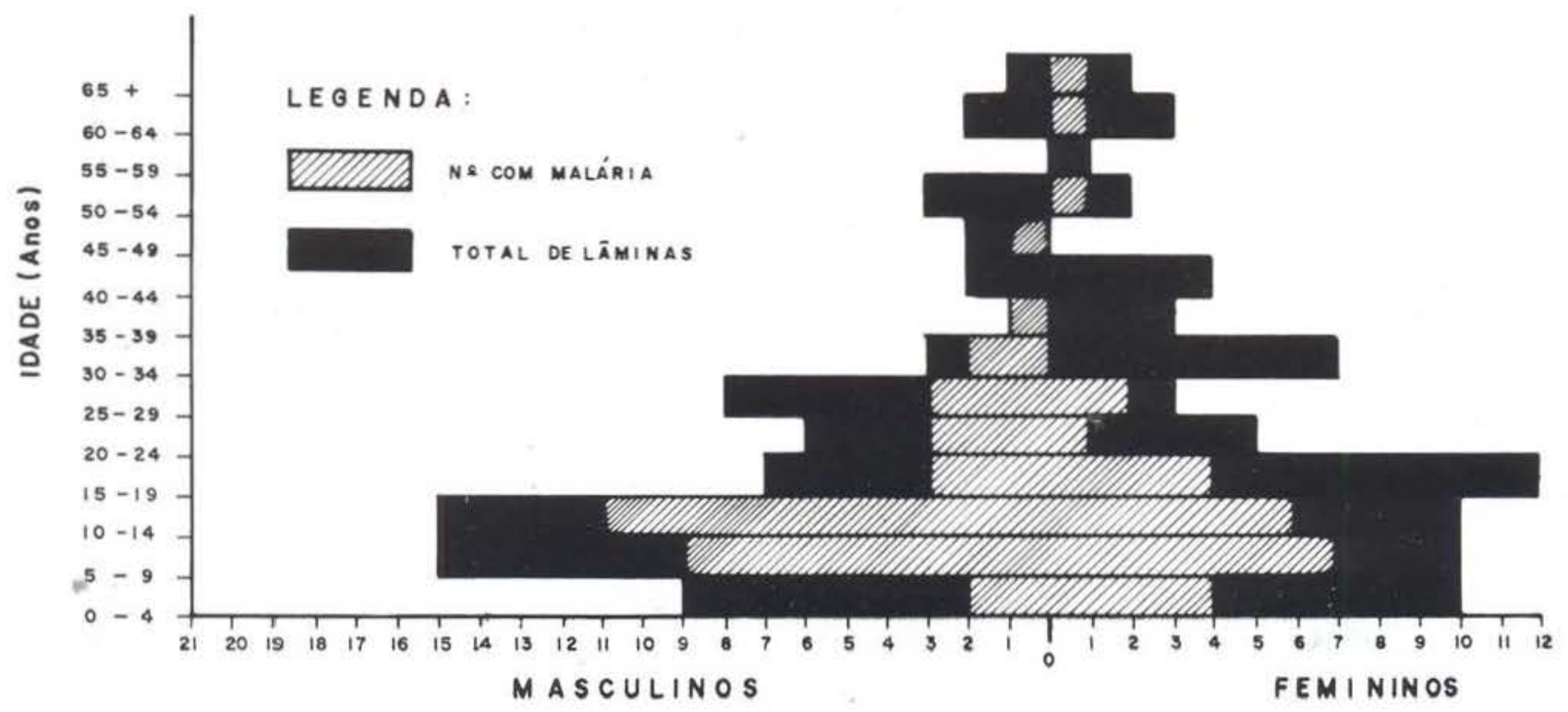

Estudo sobre... 
TABELA 02 - Tribo Mayongong total de individuos examinados, positividade por faixa etária e sexo.

\begin{tabular}{|c|c|c|c|c|c|c|c|c|c|c|c|c|c|c|}
\hline \multirow{3}{*}{$\begin{array}{l}\text { IDADE } \\
\text { (ANOS) }\end{array}$} & \multicolumn{7}{|c|}{ MASCULINO } & \multicolumn{7}{|c|}{ FEMININO } \\
\hline & \multicolumn{6}{|c|}{ PARASITEMIA } & \multirow{2}{*}{ TOTAL } & \multicolumn{6}{|c|}{ PARASITEMIA } & \multirow{2}{*}{ TOTAL } \\
\hline & $+/ 2$ & + & ++ & +++ & FG & NEG & & $+/ 2$ & + & ++ & $++t$ & FG & NEG & \\
\hline Até - 04 & & & 01 & 01 & & 07 & 09 & & & 03 & 01 & & 06 & 10 \\
\hline $05-09$ & 01 & 04 & 04 & & & 11 & 20 & 01 & 02 & 01 & & 03 & 03 & 10 \\
\hline $10-14$ & 03 & 03 & 04 & 01 & & 08 & 19 & 01 & 02 & 01 & 02 & & 04 & 10 \\
\hline $15-19$ & 01 & & 01 & & 01 & 04 & 07 & 01 & 01 & 02 & & & 08 & 12 \\
\hline $20-24$ & 01 & & 01 & & 01 & 03 & 06 & & 01 & & & & 04 & 05 \\
\hline $25-29$ & 01 & 01 & 01 & & & 05 & 08 & & 01 & & 01 & & 02 & 04 \\
\hline $30-34$ & & & & & 02 & 01 & 03 & & & & & & 07 & 07 \\
\hline $55-39$ & & 01 & & & & & 01 & & & & & & 03 & 03 \\
\hline $40-44$ & & & & & & 02 & 02 & & & & & & 04 & 04 \\
\hline $45-49$ & & & 01 & & & 01 & 02 & & & & & & & 00 \\
\hline $50-54$ & & & & & & 03 & 03 & & 01 & & & & 01 & 02 \\
\hline $55-59$ & & & & & & & 00 & & & & & & 01 & 01 \\
\hline $60-64$ & & & & & & 02 & 02 & & 01 & & & & 02 & 03 \\
\hline $65-69$ & & & & & & 01 & 01 & & & & & & 01 & 01 \\
\hline $70-74$ & & & & & & & 00 & & & 01 & & & & 01 \\
\hline $75-79$ & & & & & & & 00 & & & & & & & 00 \\
\hline $80-84$ & & & & & & & 00 & & & & & & 01 & 01 \\
\hline TOTAL & 07 & 09 & 13 & 02 & 04 & 48 & 83 & 03 & 09 & 08 & 04 & 03 & 47 & 74 \\
\hline
\end{tabular}

$+/ 2 \quad-<01$ parasita por campo mil $\times$ segundo o critério da OMS.

$+\quad 02$ parasitos por campo mil $x$ segundo o critério da OMS.

$+\quad-03$ a 20 parasitas por campo mil X segundo o critério da OMS.

+++-021 a 200 parasitas por campo mil $\times$ segundo o critério da OMS.

FG - Somente forma gametócita no sangue periférico.

NEG - Resultado Negativo.

Os dados de captura de mosquitos, durante três noites, na aldeia dos Mayongong, estão representados no gráfico III. Uma média de 64 mosquitos foi coletada por noite. Foi feita dissecção de todos os mosquitos coletados em uma noite para confirmar o vetor. Uma glâncula salivar m̌fectada e dois estômagos positivos (oocistos) de um total de 40 mosquitos, estabeleceu-se uma taxa de infecção de $8 \%$ para o Anopheles darlingi. Esta foi a única espécie de Anopheles coletada na aldeia.

Em três noites não consecutivas foram coletados mosquitos na mata próxima ao local onde os silvícolas se dirigiam para beber água. Os seguintes Anopheles foram obtidos em 9 horas/homem cie coleta: Um Anopheles nimbus, dois Anopheles oswaldoi e seis Anopheles esquamifemur. O único Anopheles coletado na aldeia foi Anopheles darlingi. 


\title{
Anopheles dorlingi
}

\author{
COLETA - ISCA HUMANA
}

ABRIL - 1.2,5/1977 - UAUARIS

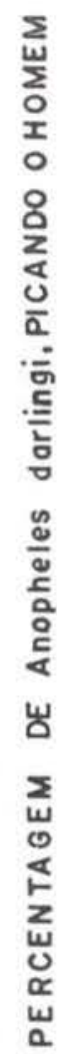

(RORAIMA)- BRASIL

TOTAL COLETADO - 193

MÉDIA POR NOITE - 64

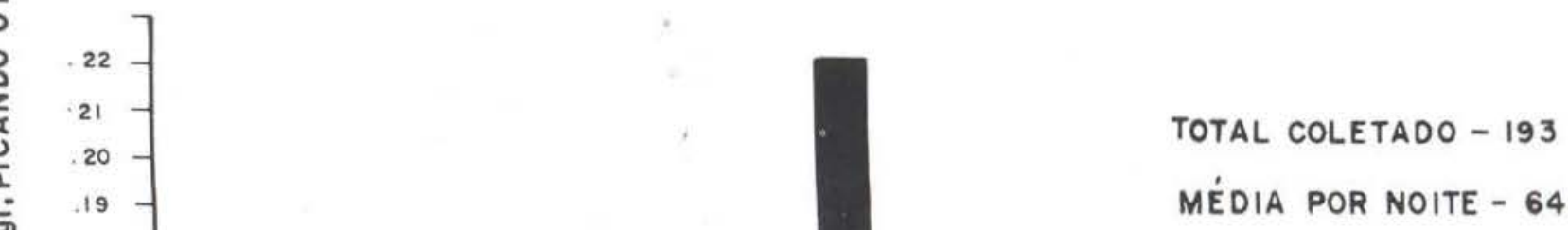

\section{Discussäo}

É interessante notar que a tribo dos Sanomã forma dois grupos geograficamente distintos: um próximo a pista de pouso e o outro vivendo temporariamente, próximo à maloca dos Mayongong. O primeiro grupo apresenta uma baixa taxa de infecção, somente 15\% (10 de 66). O segundo grupo vive praticamente com os Mayongong e apresenta uma taxa de $41 \%$ (07 de 17) (aqui o Anopheles darling foi o mais abundante), muito próxima à taxa de infecção ericontrada nos Mayongong que foi de $39 \%$ (62 de 156) .

Os missionários asseguraram-nos que nenhum surto de malária ocorreu desde 1965 e a amostra dos casos pela idade, indica que $70 \%$ são índios nascidos no ano de 1965. A tribo Sanomã apresenta uma taxa baixa de malária $21 \%$ (12 de 58 ) no sexo feminino e $31 \%$ (17 de 54) no sexo masculino. Enquanto os Mayongong apresentam uma taxa alta, 37\% (27 de 74) no sexo feminino e $42 \%$ ( 35 de 83 ) no sexo masculino. 
Um fato interessante ocorreu nessa epidemia. Um grupo dos Sanomã deixou a área uma semana antes de nossa chegada. Fomos informados pelo missionário que os índios abandonaram a aldeia para se livrarem da malária. Localizamos o grupo com ajuda do missionário e de um índio da mesma tribo, a uma hora rio acima numa canoa, com motor de popa e duas horas andando a pé no sentido oeste. O grupo estava vivendo num abrigo temporário e subsistindo em precaríssimas condições, alimentando-se de frutas e farinha de mandioca preparada antes de deixarem a aldeia central.

Uma índia de 15 anos de idade estava clinicamente enferma com malária falciparum e quatro outros apresentaram esfregaço de sangue positivos (pré-clínica). Eles tinham obviamente contraído malária entes de deixarem a aldeia central e mudaram-se para uma área onde não havia mosquitos. Nenhum Anopheles foi encontrado em 24 horas/homem de captura, naquele acampamento. Mudando-se de uma área epidémica para uma localidade livre de mosquitos (vetor) quebraria a cadeia na transmissão da malária.

\section{Tratamento}

Todos os casos de malária, nāo grave, foram tratados com cloroquina (4 aminoquinoleínas) oral ou parenteral, dependendo da natureza e idade de cada caso. Foi usado, no tratamento, o esquema padräo recomendado pela OMS (WHO, 1973). Acompanhou-se esses pacientes por um período da sete dias, com pesquisa diária da parasitemia periférica. Três casos não responderam ao tratamento pelas cloroquinas e foram tratados com sucesso usando a associação Sulfadoxina e Pirimetamina (Fansidar) em dose única oral, ou em duas doses parenteral. Em todos os casos severos usamos a mesma associação medicamentosa com ótimo resuitado.

Não houve nenhum caso fatal, apenas dois pacientes apresentavam-se em estado pré-comatose, respondendo muito bem à terapêutica. Uma mulher Mayongong faleceu um dia antes de nossa chegada; fomos informados que ela era portadora de distúrbio cárdio-respiratório, não sendo possivel esclarecer se sua morte poderia ser atribuída à malária.

\section{AGRADECIMENTOS}

Somos imensamente gratos ao missionário residente na aldeia $\mathrm{Sr}$. Paulo Silas Dinis que traduziu o Sanomã e facilitou a obtenção das amostras de sangue para os exames. A MEVA por ceder-nos uma casa que serviu de posto médico e laboratório de campo. Ao Sr. D. Borgman pela história médica pregressa da tribo e missão. Aos Srs. Fortunato Batista, Francisco Moraes de Andrade e Aitamiro Miranda Soares pela assistência técnica e, finalmente, ao Dr. Rubert Pinger que facilitou nosso acesso até o local.

\section{SUMMARY}

In april, 1977, an outbreak of falciparum malaria occured in the Sanomã and Mayongong indian tribes in Brazil (Roraima) bordering Venezuela. Two hundred and sixty-eight indians were examined for malaria and 91 cases were found. Only one vector species, Anopheles darlingi was found, whose activities averaged 64 bites per man per night. Three cases of falciparum were resistant to chloroquine but responded favorably to Fansidar (sulphadoxina and pyrimethamine).

There were aistinct differences between tribes based on the proximity to the vector population. These differences are presented by age and sex. One group of Sanomã in an effort to escape malaria moved to a remote area, which interestingly was free of man-biting mosquitoes.

\section{BIBLIOGRAFIA CITADA}

Borgman, DONALD

$$
\begin{aligned}
& 1977 \text { - Comunicaçāo pessoal - (MEVA), C.P. } \\
& \text { 209, Boa Vista-Roraima. }
\end{aligned}
$$

Organização panamericana da Saúde/Organtzação Mundial da Saúde

1975 - Publicação científica n.• 276.

WILBERT, JOHANNES

1963 - Indios de la Region Ormoco-Venturari. - Fundacion La Sale de Ciencias $\mathrm{Na}$ turales, - Monografia n. 8 - Caracas.

World Health ORganization

1973 - Chemotherapy of malaria and Resistance to Antimalarials. Tech. Rep. n. 529. 